\title{
Protective effect of ultrasound microbubble combined with gross saponins of tribulus terrestris on glaucomatous optic nerve damage
}

\author{
Xiaoli Shen ${ }^{1 \#}$, Junhong Guo ${ }^{1 \#}$, Ning Fan ${ }^{1}$, Mingying Lai ${ }^{1}$, Lina Huang ${ }^{1}$, Jiantao Wang ${ }^{1}$, Qiang Li $^{2}$ \\ ${ }^{1}$ Department of Glaucoma, Shenzhen Eye Hospital, Shenzhen Eye Hospital Affiliated with Jinan University, School of Optometry, Shenzhen \\ University, Shenzhen, China; ${ }^{2}$ Department of Ophthalmology, Shenzhen People's Hospital (The Second Clinical Medical College, Jinan University, \\ The First Affiliated Hospital, Southern University of Science and Technology), Shenzhen, China \\ Contributions: (I) Conception and design: X Shen, J Guo; (II) Administrative support: Q Li, J Wang; (III) Provision of study materials or patients: N \\ Fan; (IV) Collection and assembly of data: M Lai; (V) Data analysis and interpretation: L Huang; (VI) Manuscript writing: All authors; (VII) Final \\ approval of manuscript: All authors. \\ "These authors contributed equally to this work. \\ Correspondence to: Qiang Li. Department of Ophthalmology, Shenzhen People's Hospital (The Second Clinical Medical College Jinan University, The \\ First Affiliated Hospital, Southern University of Science and Technology), Shenzhen 518020, China. Email: gemmyleon@163.com; Jiantao Wang. \\ Shenzhen Eye Hospital, Shenzhen Eye Hospital Affiliated with Jinan University, School of Optometry, Shenzhen University, Shenzhen 518040, \\ China. Email: wangjiantao65@126.com.
}

Background: To investigate the protective effect of ultrasound microbubble combined with gross saponins of tribulus terrestris (GSTT) (a Chinese herb) on glaucomatous optic nerve damage.

Methods: Rabbits were randomly divided into five groups. Normal (Group A), high intraocular pressure (IOP, Group B), GSTT (Group C), GSTT + ultrasound (Group D), and GSTT + ultrasound + microbubble destruction (Group E). The high intraocular pressure eye (model eye) was compared to the normal eye (control eye) at 1, 2, and 4 weeks after model establishment. Rabbits were sacrificed 4 weeks later to measure the retina thickness using Cirrus OCT, slit lamp photograph, and fundus photography. The retina and optic nerve of rabbits in each group were collected and the stretched retina were prepared for retinal ganglion cell (RGC) counting, the optic nerve axon was measured, and a transmission electron microscopy was used.

Results: Retina thickness based on Cirrus OCT: mean retinal thickness in Group E was significantly greater than that in Group B, but still thinner than that in Group A. RGCs counts: RGCs counts in Group E were significantly higher than those in Groups B, C, and D but still lower than those in Group A. Quantitative analysis of optic nerve axons: In Group E, the number of optic nerves was increased, diameters of optic nerve axons were decreased, the percentage of optic nerve area occupied by axons was increased, and there were statistically significant differences compared to Groups B, C, and D. Content of GSTT in retina: The content of GSTT in Group E was significantly higher than that in other groups. Observation of the rabbit optic nerves: In Group E, the structure of the myelin sheath of the optic nerve was still intact but less ordered, and the microtubule and microfilament structures in the axons were clear.

Conclusions: Combination of the ultrasound microbubble and GSTT can improve the protective effect of GSTT on optic nerve damage in rabbits with ocular hypertension.

Keywords: Ultrasound microbubble; gross saponins of tribulus terrestris (GSTT); optic nerve injury; rabbit

Submitted Jul 16, 2021. Accepted for publication Sep 09, 2021.

doi: $10.21037 / \mathrm{atm}-21-4230$

View this article at: https://dx.doi.org/10.21037/atm-21-4230 


\section{Introduction}

Glaucoma is an optic neuropathy characterized by increased intraocular pressure (IOP) as the main risk factor, and apoptosis of retinal ganglion cells (RGCs) is the main pathological feature. Apoptosis leads to the death of RGCs after intraocular hypertension, autophagy and accessory apoptosis may also be involved in the death of RGCs. Lowering of increased intraocular pressure is no longer the only treatment for glaucoma, and optic nerve protection has become a major research focus in the drug treatment of glaucoma worldwide (1). Traditional Chinese herbal medicine has shown unique therapeutic effects for various diseases and disorders with the advantages of few side-effects and low cost. At present, the more extensively studied herbs include salvia miltiorrbiza, erigeron breviscapus, ginkgo biloba, and puerarin. Tribulus terrestris is an annual herb, which belongs to the zygophyllaceae family. The main active phytoconstituents of this plant include flavonoids, alkaloids, saponins, lignin, amides, and glycosides. The plant parts have different pharmacological actions including aphrodisiac, anti-inflammatory, antimicrobial, and antioxidant actions. Tribulus terrestris is used clinically to treat diseases including Alzheimer's disease, eye disease, hypertension, cardiovascular diseases, diabetes, infertility, impotence, and ischemic stroke. It has been shown to have a neuroprotective effect (2-4).

It is well-known that optic nerve protective drugs can be administered orally, intravenously, and through intramuscular injection; in each case the drugs first enter the systemic circulation before reaching the ocular tissues, leading to an unsatisfactory therapeutic effect of optic nerve protective drugs in clinical practice. Therefore, there is an urgent need for novel administration modes that can make optic nerve protective drugs more effective and safer for local application to the retina and optic nerve. As a new type of drug transfer carrier, ultrasound microbubble produces a variety of biological effects after ultrasound irradiation, such as cavitation and sonoporation effects, and it produces some reversible pores of different sizes and numbers on the surface of cell membranes. This effect increases the local capillary and cell membrane permeability, such that the drug can more easily enter the tissue cells and undergo a directional release in the target tissue to achieve the goal of targeted therapy (5). However, there are no studies combining ultrasound microbubble with traditional Chinese medicine gross saponins of tribulus terrestris (GSTT) to treat optic nerve damage in ocular hypertension. In this study, a rabbit model of optic nerve injury induced by ocular hypertension was established, and then, ultrasound microbubble was combined with GSTT to investigate the protective effect of this new drug administration mode combined with GSTT on glaucomatous optic nerve damage. We present the following article in accordance with the ARRIVE reporting checklist (available at https:// dx.doi.org/10.21037/atm-21-4230).

\section{Methods}

\section{Subjects}

Male and female New Zealand white rabbits with a body weight of $1.5 \mathrm{~kg}$ were purchased from Guangdong Medical Laboratory Animal Center. The animals were raised in the animal facility of the Medical School of Shenzhen University, and the maintenance conditions were clean and well-ventilated conditions, and constant temperature and humidity. The animals were kept in individual cages with mixed feed. Experiments were performed under a project license granted by the Ethics Committee of Medical School of Shenzhen University, in compliance with the International Association of Veterinary Editors' Consensus Author Guidelines on Animal Ethics and Welfare for the care and use of animals.

\section{Grouping of experimental animals}

Forty healthy New Zealand white rabbits were randomly divided into five groups, with eight rabbits in each group, which were maintained under specific-pathogen-free (SPF) condition. All authors were aware of the group allocation at the different stages of the experiment. The normal control group (Group A) received no treatment. For the high intraocular pressure model group (Group B), a high intraocular pressure model only was established. Group $\mathrm{C}$ received high intraocular pressure model + intravitreal injection of GSTT group, with establishment of high intraocular pressure model and intravitreal injection of $0.1 \mathrm{~mL}$ GSTT solution $(7.5 \mathrm{mg} / 0.1 \mathrm{~mL})$ alone. Group D received intraocular pressure model + intravitreal injection of GSTT + ultrasound group, whereby high intraocular pressure was established and intravitreal injection of GSTT solution $(7.5 \mathrm{mg} / 0.1 \mathrm{~mL})$ was administered. The rabbit eye was closed, the coupling agent was applied, the ultrasound probe was placed over the eyeball, and it was immediately irradiated with a frequency of $1 \mathrm{MHz}$ and a sound intensity of $0.5 \mathrm{~W} / \mathrm{cm}^{2}$ for $60 \mathrm{~s}$. Group E received high intraocular pressure model + intravitreal injection of 

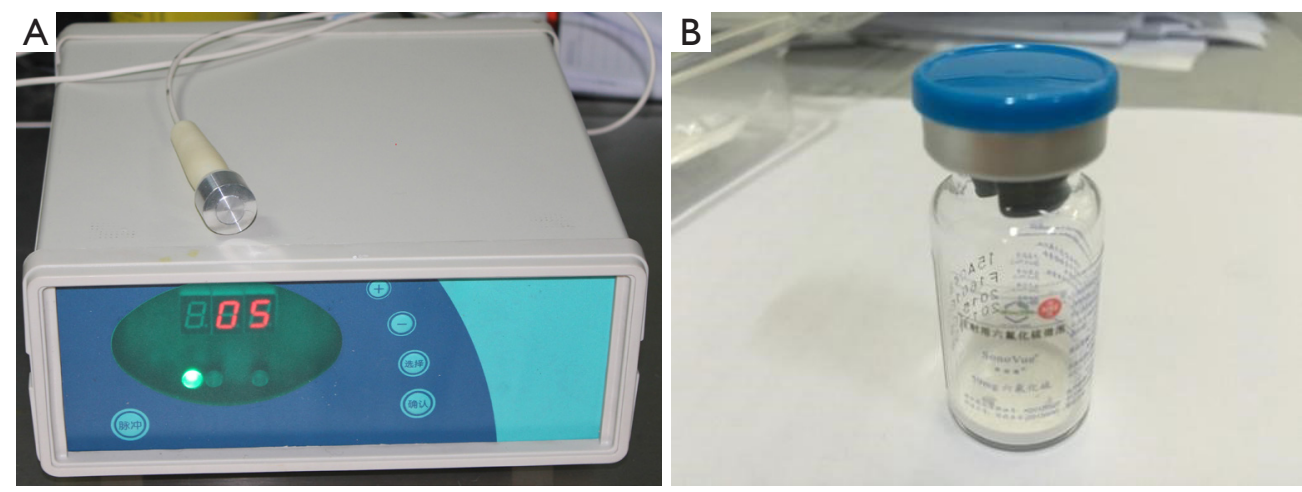

Figure 1 Main reagent and instrument. (A) Ultrasonic gene transfection therapeutic apparatus; (B) microbubble suspension.

GSTT + ultrasound + microbubble group, whereby high intraocular pressure was established, an intravitreal injection of GSTT solution $(7.5 \mathrm{mg} / 0.1 \mathrm{~mL})$ was administered and microbubble suspension was applied. The rabbit eye was closed, the coupling agent was applied, and then ultrasound irradiation with the above parameters was applied. The above procedures were performed once a week for three weeks in each group.

\section{Establishment of high intraocular pressure model}

The animals were anesthetized with an intravenous injection of $3 \%$ pentobarbital sodium $(1 \mathrm{~mL} / \mathrm{kg})$ into the marginal ear vein. After topical ocular anesthesia with Alcaine eye drops, an eye speculum was used to open the eyelid, and $0.2 \mathrm{~mL}$ of anterior chamber aqueous humor was withdrawn at $1 \mathrm{~mm}$ inside the corneoscleral limbus at the 9 o'clock position with a $1 \mathrm{~mL}$ syringe. Then, $0.2 \mathrm{~mL}$ of $0.3 \%$ compound carbomer solution was injected into the anterior chamber at $1 \mathrm{~mm}$ inside the corneoscleral limbus on the contralateral side. After the surgery, the eyes were treated with Tarivid eye drops. If the intraocular pressure was found to be $<22 \mathrm{mmHg}$ during the experimental period, the injection was repeated once 7 days later according to the above method. The optic nerve injury model in rabbits with high intraocular pressure was based on the criteria that the intraocular pressure was $>22 \mathrm{mmHg}$ and that the pressure was maintained for 4 weeks (6). Intraocular pressure was measured and recorded daily at the same time using a TonoPen tonometer (Reichert, Depew, NY, USA).

\section{Main reagents and instruments}

Microbubbles (Bracco, Milan, Italy), gross saponin powder of tribulus terrestris (purity $\geq 95 \%$ ) (National Institutes of Food and Drug Control, China), Carbomer-940 (Guoren Yikang Technology Co., Ltd., Beijing, China), a visual electrophysiology system (ROLAND Germany), Cirrus OCT (Zeiss, Oberkochen, Germany), a light microscope (Olympus, Tokyo, Japan), high performance liquid chromatography (HPLC) (Shimadzu Corporation, Kyoto, Japan), and a transmission electron microscope (FEI, Hillsboro, OR, USA) were used.

The ultrasonic gene transfection therapeutic apparatus used in this experiment was purchased from the Institute of Ultrasonic Imaging, Chongqing Medical University. Its center frequency is $1 \mathrm{MHz}$, the diameter of its probe is $1 \mathrm{~cm}$, and its adjustable sound intensity range is 0 to $3.0 \mathrm{~W} / \mathrm{cm}^{2}$. It has both continuous and pulsed emission modes, and the emission time can be set to be accurate to the second (Figure 1A).

Preparation of microbubble suspension: Five millilitre of $0.9 \%$ physiological saline was slowly injected into the SonoVue vial (Bracco, Bucks, UK) containing the lyophilized powder, which was then allowed to stand for subsequent use. Before use, the vial was shaken vigorously to generate microbubbles. The microbubble concentration was $2 \times 10^{8} / \mathrm{mL}$, and the average microbubble diameter was $2.5 \mu \mathrm{m}$. The prepared microbubble solution was used within $6 \mathrm{~h}$ (Figure 1B).

\section{Detection indicators}

\section{Cirrus OCT examination}

Compound tropicamide eye drops $(0.5 \%)$ were applied once every five min. a total of three times, and the head of the rabbit was fixed in an appropriate position with mandibular rest using Cirrus OCT after full mydriasis. The maxillary or 
mandibular rest was adjusted so that the optical coherence tomography (OCT) detection light source was aligned with the retina at $1 \mathrm{PD}$ right below the optic papilla (6 o'clock position) by observing it on the monitor, the 4-mm linear scan mode was used, and the detection was repeated at least four times. Images with a good scanning position and a signal intensity $\geq 5$ were saved to disk. Cirrus OCT was used to automatically identify the retinal pigment epithelium layer and the internal limiting membrane. The operator equally divided the OCT scan image into four parts in the horizontal direction and manually measured the thickness of the neuroepithelial layer of the retina at the three sites in the middle using the scale provided by the computer; the average value was recorded as the mean retinal thickness $(\mu \mathrm{m})$.

\section{Histology examination}

After 4 weeks, the rabbits were sacrificed. The retina and optic nerve were dissected and rabbit optic nerves were stretched for optic nerve axon measurement, retinal ganglion cells (RGC) were counted, and a transmission electron microscopy was used (TEM).

\section{High performance liquid chromatograph examination}

The contents of GSTT in rabbit retina were detected using HPLC.

\section{Statistical analyses}

The Statistical Program for the Social Sciences Version 16.0 software was used for statistical analysis, and oneway analysis of variance was used to compare data between multiple groups. For pairwise comparisons, the StudentNewman-Keuls (SNK) method was used when the variances were homogeneous, and Tamhane's method was used when the variances were heterogeneous. Differences were considered statistically significant if $\mathrm{P}<0.05$.

\section{Results}

\section{Slit lamp and fundus photography}

Compared to Group A (Figure 2A), in Group B, congestion of the conjunctiva was obvious, there was conjunctiva vascular tortuous dilation and corneal fog edema, iris texture was not clear, the pupil was loose and fixed, the lens was still transparent, and the remaining details were not clear (Figure 2B).

Compared to Group A (Figure 2C), in Group B, the optic disc was edematous, the boundary was not clear, and it turned pale; the cup was obviously enlarged (Figure 2D).

\section{Intraocular pressure measurement}

As shown in Table 1, the IOP in the model eye was $15.0 \pm 2.0 \mathrm{mmHg}$, and the IOP in the control eye was $13.6 \pm 1.5 \mathrm{mmHg}$; there was no significant difference in the IOP between the control and the model eye $(\mathrm{P}=0.137)$. After modeling, the IOP in the model eye $(33.4 \pm 2.8,34.1 \pm 2.5$ and $34.8 \pm 2.2 \mathrm{mmHg}$ ) was significantly different from that of the control eye $(13.6 \pm 1.8,13.4 \pm 1.7$ and $13.3 \pm 1.4 \mathrm{mmHg})$ at 1,2 and 4 weeks $(\mathrm{P}<0.001)$.

\section{Comparison of rabbit retinal thickness measured using Cirrus OCT}

As shown in Table 2, the difference in the mean retina thickness of rabbits in each group was statistically significant $(\mathrm{P}<0.001)$. Pairwise comparisons and analyses showed that the mean retina thickness in Group B was significantly thinner than that in Group A $(\mathrm{P}<0.05)$ (Figure 3). The mean retina thickness in Group E was significantly greater than that in Group B but still thinner than that in Group A, and the difference was statistically significant $(\mathrm{P}<0.05)$.

However, the difference in the mean retina thickness in Groups $\mathrm{C}, \mathrm{D}$, and $\mathrm{E}$ was not statistically significant $(\mathrm{P}>0.05)$.

\section{RGCs count in the retina of rabbits measured using light microscopy}

As shown in Table 3, the differences in the RGCs counts for each group were statistically significant $(\mathrm{P}=0.002)$. Pairwise comparisons and analyses showed that the RGCs count in Group B was significantly smaller than that in Group A $(\mathrm{P}<0.05)$. The RGCs count in Group E was significantly larger than that in Groups B, C, and D, but still lower than that in Group A $(\mathrm{P}<0.05)$. However, the RGCs count was not significantly different compared with Groups $\mathrm{C}$ and $\mathrm{D}$ $(\mathrm{P}>0.05)$.

\section{Quantitative analysis of rabbit optic nerve axons using light microscopy}

Using Nissl staining of the rabbit optic nerve, the nucleus was light blue, and the neurons contained abundant Nissl bodies and an intracellular alkaliphilic substance that was dark blue and in the form of plaques or granules. In Group 

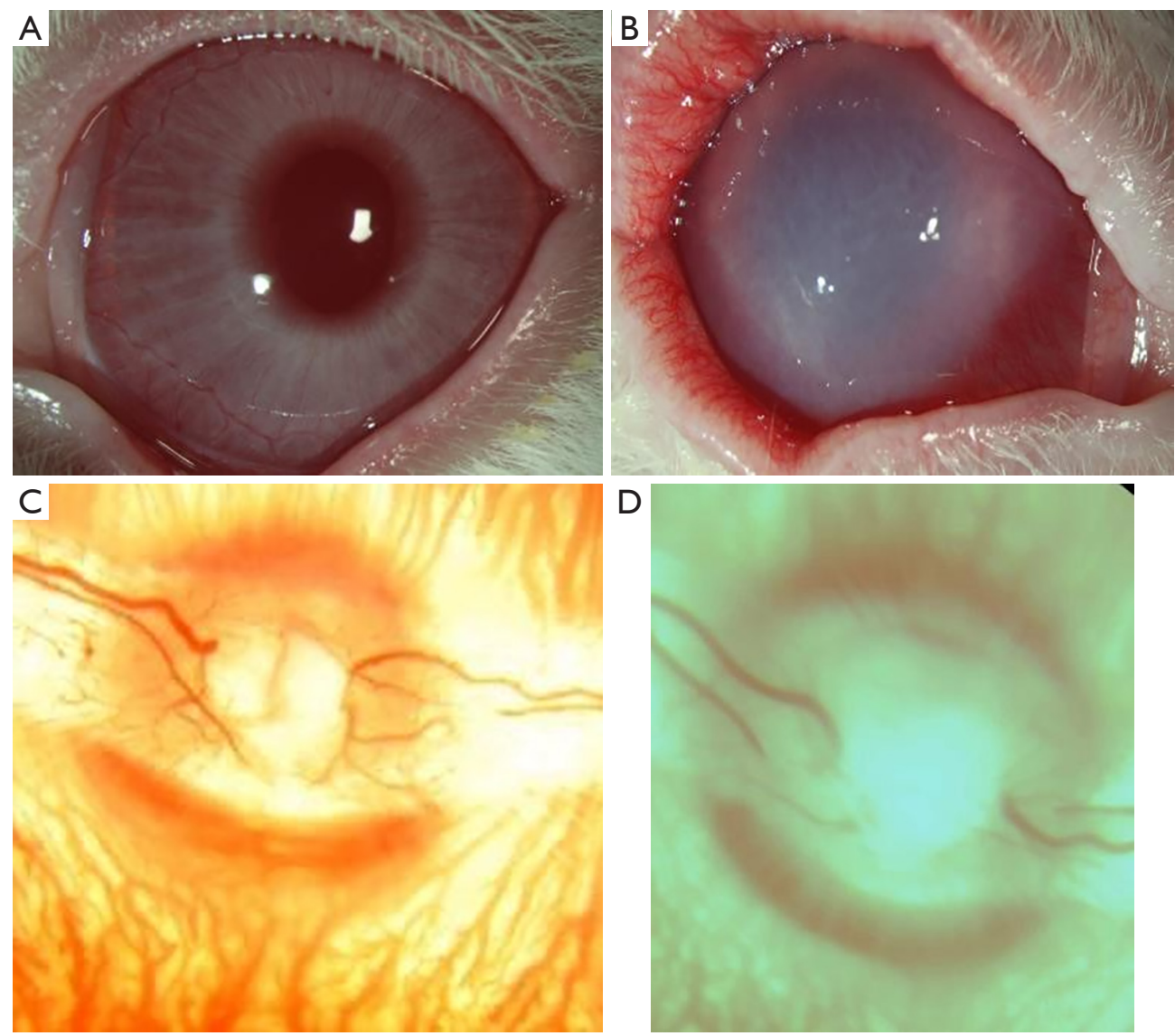

Figure 2 Slit lamp and fundus photography. (A) Group A; (B) Group B; (C) Group A; (D) Group B.

Table 1 Comparison of intraocular pressures between the control eye and the model eye at different times $(\bar{x} \pm s, \mathrm{mmHg})(7)$

\begin{tabular}{lcccc}
\hline Group & Before modeling & 1 week & 2 weeks & 4 weeks \\
\hline Control eye & 13.61 .5 & 13.61 .8 & 13.41 .7 & 13.31 .4 \\
Model eye & 15.02 .0 & 33.42 .8 & 34.12 .5 & 34.82 .2 \\
$\mathrm{t}$ & -1.561 & -17.845 & -21.308 & -22.71 \\
$\mathrm{P}$ & 0.137 & 0.000 & 0.000 & 0.000 \\
\hline
\end{tabular}

Independent samples $t$-tests. This table can be read in our published paper (7).

A, more Nissl bodies were stained in dark blue, and thick optic nerve axons were observed using light microscopy (Figure 4A,4B).

As shown in Table 4, the differences in the number of optic nerve axons, the diameter of optic nerve axons, and the percentage of the optic nerve area occupied by axons among rabbits in different groups were significantly different $(\mathrm{P}=0.000,0.001$ and 0.02 , respectively). Pairwise
Table 2 Comparisons of rabbit retinal thickness measured using Cirrus OCT $(\bar{x} \pm s)$

\begin{tabular}{lcc}
\hline Group & Number of eyes & Retinal thickness $(\mu \mathrm{m})$ \\
\hline Group A & 8 & 226.204 .49 \\
Group B & 8 & 194.005 .40 \\
Group C & 8 & $203.852 .09^{\triangle}$ \\
Group D & 8 & $205.602 .18^{\Delta}$ \\
Group E & 8 & $211.852 .04^{\Delta}$ \\
P & & 0.000 \\
\hline
\end{tabular}

One-way analysis of variance, ${ }^{\Delta}$ indicates that there was no statistically significant difference in pairwise comparisons between groups $(P>0.05)$, and that there was a statistically significant difference in pairwise comparisons between the remaining groups $(\mathrm{P}<0.05)$.

comparison and analysis showed that in Group B, the optic nerve was swollen, the number of optic nerves was reduced, the diameter of the optic nerve axons increased, 

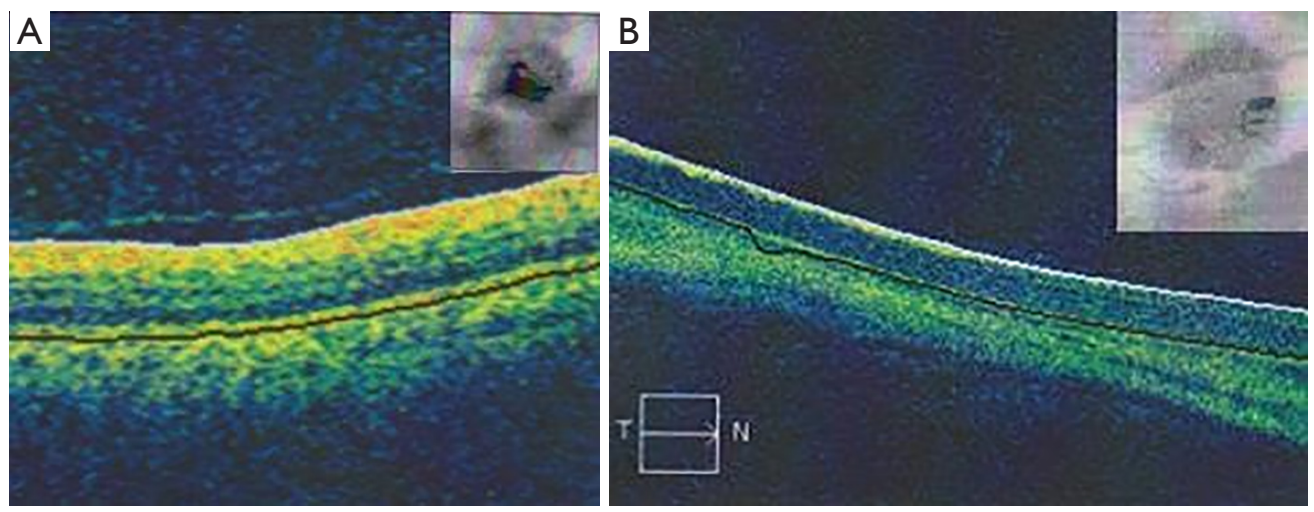

Figure 3 Comparison of rabbit retinal thickness measured by Cirrus OCT. (A) Group A; (B) Group B (the retina thickness was significantly thinner).

Table 3 Comparison of retinal ganglion cell (RGCs) counts using light microscopy $(\bar{x} \pm s)$

\begin{tabular}{lc}
\hline Group & Number of retinal ganglion cells \\
\hline Group A & 26.040 .70 \\
Group B & $14.971 .30^{\triangle}$ \\
Group C & $18.980 .86^{\Delta}$ \\
Group D & $19.650 .80^{\triangle}$ \\
Group E & $23.870 .72^{\circ}$ \\
P & 0.002 \\
\hline
\end{tabular}

One-way analysis of variance, ${ }^{\Delta}$ indicates that there were no statistically significant differences in pairwise comparisons between groups $(P>0.05)$, and there was a statistically significant difference in pairwise comparisons between the remaining groups $(\mathrm{P}<0.05)$. the percentage of optic nerve area occupied by axons was decreased, and there was a statistically significant difference compared with Group A $(\mathrm{P}<0.05)$. After intravitreal injection of GSTT, and with the application of ultrasound combined with microbubbles the number of optic nerves was increased in Group E, the diameter of optic nerve axons was decreased, the percentage of optic nerve area occupied by axons was increased, and there was a statistically significant difference compared with Groups B, C, and D $(\mathrm{P}<0.05)$

\section{Content of GSTT in rabbit retina was determined using HPLC}

The results of standard product chromatogram of GSTT
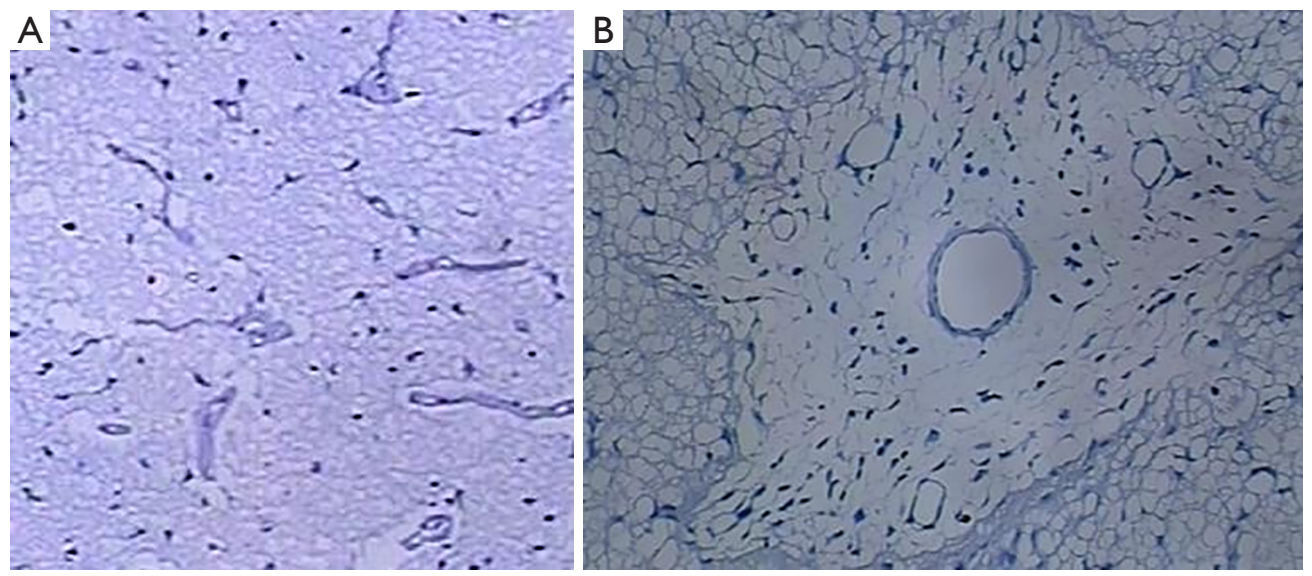

Figure 4 Nissl bodies and optic nerve axons of rabbit in Group A. (A) Dark blue granules, Nissl bodies are visible in Group A; (B) Rabbit optic nerve axons in Group A. HE, ×200. 
Table 4 Quantitative analysis results of the rabbit optic nerve axons

\begin{tabular}{|c|c|c|c|}
\hline Group & \multicolumn{3}{|c|}{ Quantitative index of optic nerve axon $(x \pm s)$} \\
\hline Group B & 104.0035 .27 & 1.3230 .049 & 34.602 .63 \\
\hline Group C & 176.5032 .66 & 1.0310 .015 & $45.052 .09^{\Delta}$ \\
\hline Group E & 205.2030 .18 & 1.1350 .029 & 49.952 .02 \\
\hline$P$ & $<0.001$ & 0.001 & 0.02 \\
\hline
\end{tabular}

One-way analysis of variance, ${ }^{\Delta}$ indicates that there were no significant differences in pairwise comparisons between groups $(P>0.05)$, and there was a statistically significant difference in pairwise comparisons between the remaining groups $(P<0.05)$.

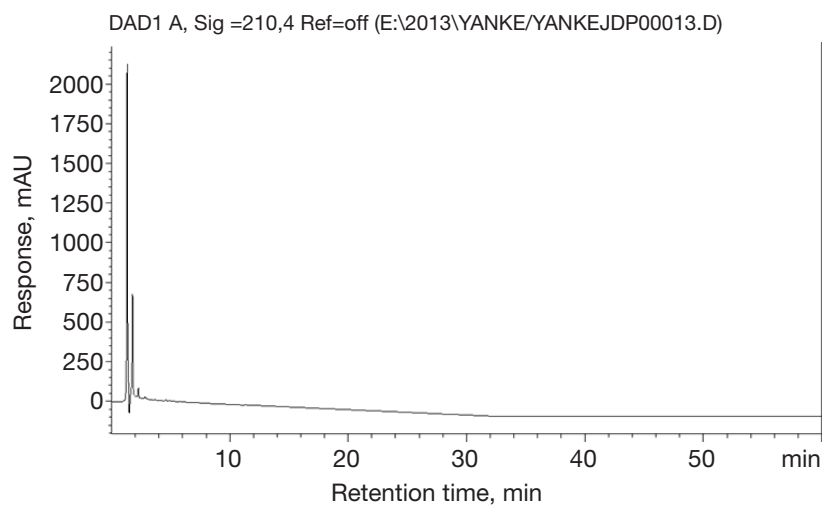

Figure 5 Standard product chromatogram of gross saponins of tribulus terrestris.

Table 5 The content of gross saponins of tribulus terrestris (GSTT) in rabbit retina was determined by high performance liquid chromatography

\begin{tabular}{lc}
\hline Group & GSTT $(\bar{x} \pm \mathrm{s}, \mathrm{mg} / \mathrm{mL})$ \\
\hline Group A & - \\
Group B & - \\
Group C & $1.630 .42^{\Delta}$ \\
Group D & $1.930 .51^{\Delta}$ \\
Group E & 3.060 .69 \\
P & 0.000 \\
\hline
\end{tabular}

One-way analysis of variance, ${ }^{\Delta}$ indicates that there were no significant differences in pairwise comparisons between groups $(P>0.05)$. are shown in Figure 5. As shown in Table 5, the content of GSTT in each group was statistically significant $(\mathrm{P}<0.001)$.

After pairwise comparison and analysis, there were no statistically significant differences in the content of GSTT in Groups $\mathrm{C}$ and $\mathrm{D}(\mathrm{P}>0.05)$. However, the content of GSTT in Group E was significantly higher than that in other groups, and this difference was statistically significant $(\mathrm{P}<0.05)$.

\section{The ultrastructure of optic nerve using transmission electron microscopy (Figure 6)}

Group A: optic nerve axons were regular, and the structure of myelin sheaths was intact; microtubule and microfilament structures were clearly visible in the axoplasm.

Group B: the optic nerve myelin sheath was dissolved and loose; axon structure was irregular, and microtubule and microfilament structures disappeared.

Group C: optic nerve axons were irregular in size and disorganized in arrangement, and some myelin sheaths were thin, loose, and detached; microtubules and microfilaments in the axons were swollen but did not disappear.

Group D: the myelin sheath of the optic nerve axons was thin, and its arrangement was disordered and loose; microtubules and microfilaments were visible in the axons.

Group E: the optic nerve myelin sheath structure was intact, and the arrangement was dense but less ordered; microtubule and microfilament structures were clear in the axons. 


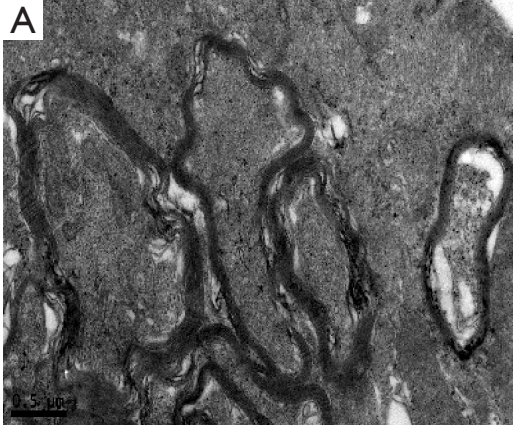

Group A: The structure of the myelin sheath was intact, and the microtubules and microfilaments were visible $(\times 26,500)(7)$

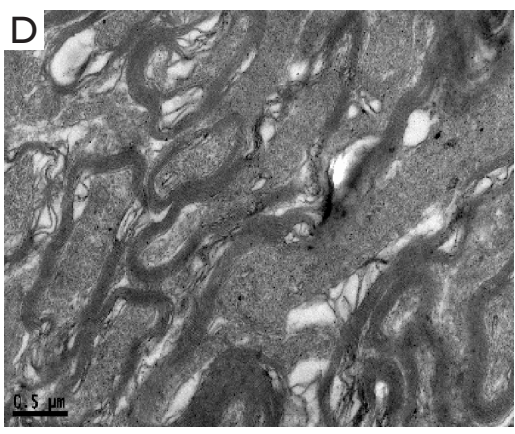

Group D: The arrangement of the myelin sheath was disordered and loose $(\times 26,500)$

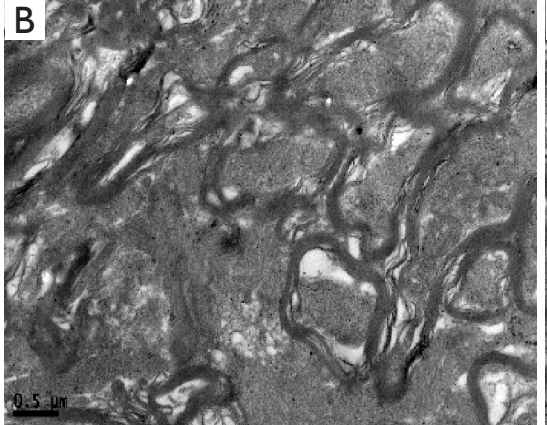

Group B: Axon structure was disordered, and the microtubule and microfilament structures disappeared $(\times 18,500)$

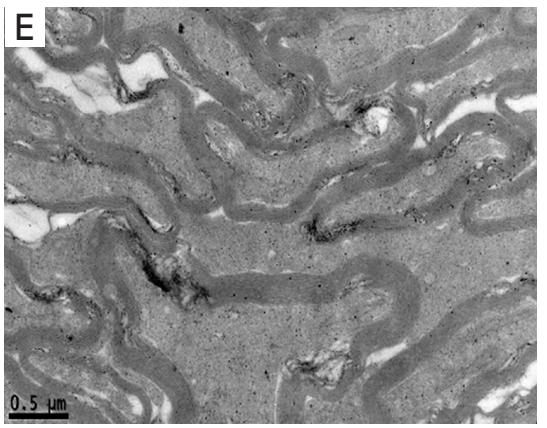

Group E: The arrangement of the myelin sheath was dense with microtubules and microfilaments $(\times 26,500)$

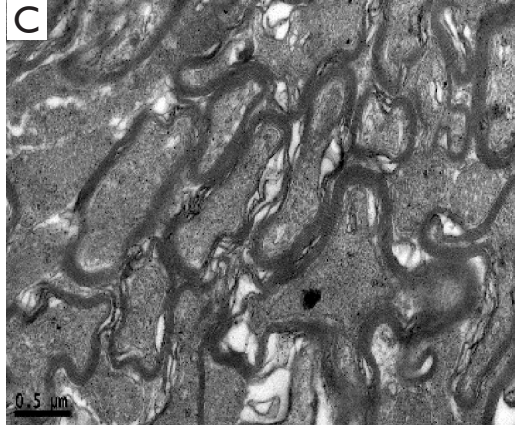

Group C: Optic nerve axons were irregular some myelin sheaths were thin, loose, and detached $(\times 46,000)$

Figure 6 Ultrastructure of the rabbit optic nerve in each group.

\section{Discussion}

SonoVue is a new type of microbubble produced by Bracco, Italy, of which the outer membrane is a lipid membrane that encapsulates sulfur hexafluoride inside. Approximately $90 \%$ of the microbubbles have a diameter less than $8 \mu \mathrm{m}$. It is relatively stable in blood vessels and can pass through the alveolar microcirculatory barrier and can be excreted from the body through the pulmonary circulation (8). Many studies have confirmed that microbubble combined with ultrasound can provide an effective and safe drug delivery method for the treatment of neurodegenerative diseases $(9,10)$. Glaucoma is essentially a neurodegenerative disease of neurons in the central nervous system, with many features similar to Alzheimer's and Parkinson's disease. Therefore, local ocular administration of drugs carried by ultrasound microbubble provides a new approach for the treatment of glaucomatous optic nerve damage. In our previous research, we have screened and determined the ideal ultrasonic parameters (ultrasonic intensity of $0.5 \mathrm{~W} / \mathrm{cm}^{2}$, irradiation time of $60 \mathrm{~s}$ ) for the rabbit retina, which provided the experimental basis for this study (7).

To date, GSTT has been the focus and a popular topic of research. It can reduce the degree of cardiomyocyte injury caused by ischemia-reperfusion by increasing the endogenous antioxidant capacity of the body and decreasing the oxidation of lipids, thus exerting a direct protective effect on cardiomyocytes $(3,11,12)$. A previous study found that GSTT could increase the survival rate of rat RGCs cultured in vitro and had the effect of promoting the growth of RGCs in mixed culture (13). In a rabbit model of chronic ocular hypertension, the protective effects of GSTT and erigeron breviscapus injection on RGCs in rabbits with chronic ocular hypertension were observed, and the study results revealed that GSTT could effectively reduce RGCs injury and had a certain protective effect (14). Both in vitro and in vivo, GSTT has a good protective effect on RGCs, and it is a highly promising optic nerve protective drug.

In this study, a compound carbomer-induced rabbit glaucoma model was used. Results showed that the average intraocular pressure was $33 \mathrm{mmHg}$ at 1 week (Table 1). After model establishment, and the high intraocular pressure 
lasted for 4 weeks. This method is easy to operate and control, and as such it is an ideal model for studies involving glaucomatous optic nerve damage. Cirrus OCT enables high-resolution tomographic imaging of ocular tissues with a resolution of 5 to $10 \mu \mathrm{m}$, allowing quantitative tracking of subtle changes in tissue structure during the course of the disease. In this study, the measurement of the retina thickness of rabbits using Cirrus OCT showed that the retina was significantly thicker in the intravitreal injection of GSTT group than the high intraocular pressure model group $(\mathrm{P}<0.05)$, indicating that the intravitreal injection of GSTT played a certain protective role against optic nerve damage caused by high intraocular pressure. There was no statistically significant difference in retina thickness measured using Cirrus OCT between the ultrasound microbubble combined with intravitreal injection of GSTT group and the intravitreal injection of GSTT alone group $(\mathrm{P}>0.05)$, likely because Cirrus OCT measurement of retina thickness is a manually assisted measurement method that requires the naked eye to identify the internal limiting membrane and retinal pigment epithelium layer when the computer makes line drawing errors while automatically identifying the internal limiting membrane and retinal pigment epithelium layer, leading to large errors in the detection results.

The results of our study showed that RGCs counting in the retina of rabbits in Group B was significantly reduced, but RGCs counting in Group E was significantly increased, and there was a statistically significant difference compared with other groups $(\mathrm{P}<0.05)$. At the same time, under high intraocular pressure, the rabbit optic nerve axon structure was disturbed, the myelin sheath was dissolved and loose to various degrees, and microtubule and microfilament structures disappeared. After intravitreal injection of GSTT, the number of optic nerve axons increased, the diameter of optic nerve axons decreased, and the percentage of the cross-sectional area of the optic nerve occupied by the optic nerve axon increased. When ultrasound was combined with microbubbles in the intravitreal injection of GSTT group, the optic nerve axon swelling, axon diameter, and the degree of myelin sheath loss of optic nerve axon were significantly reduced, and the number of axons was significantly increased $(\mathrm{P}<0.05)$. This outcome also indicated that ultrasound microbubble could significantly increase the protective effect of GSTT on RGCs and optic nerve in the retina with high intraocular pressure. In addition, in this study, the optic nerve ultrastructure was observed. The results of our study showed that the rabbit optic nerve axon structure was disturbed, the myelin sheath was dissolved and loose to various degrees, and the microtubule and microfilament structures disappeared under the high intraocular pressure. In Group E, the myelin sheaths of the optic nerves were densely and neatly arranged, and structures such as microtubules and microfilaments were visible. This outcome indicates that ultrasound microbubble combined with GSTT not only had a protective effect on RGCs injuries induced by high intraocular pressure but also played a role in the protection of the rabbit optic nerve. In addition, the content of GSTT in rabbit retina was determined by HPLC. The results showed that the differences in content of GSTT in each group were statistically significant $(\mathrm{P}<0.001)$. The content of GSTT in Group E was significantly higher than that in the other groups, and the difference was statistically significant $(\mathrm{P}<0.05)$.

Recently, great progress has been made in the study of carrying genes or drugs using ultrasound microbubble (15-17). Lin (18) investigated the safety of ultrasoundaided microbubble exposure and the association between temporal changes in round window membrane permeability and ultrastructure, and the results showed that ultrasoundaided microbubble appear to be safe and effective for inner ear drug delivery. The mechanism of enhanced permeability may involve a disruption of the continuity of the outer round window membrane epithelial layer, which controls transmembrane transport of various substances. Fu (19) established a clamped optic nerve injury model in SD rats to observe the protective effect of ultrasound microbubblemediated simultaneous transfection of retinal and visual cortex cells with brain-derived neurotrophic factor (BDNF) on RGCs after optic nerve injury, and the results showed that the number of surviving RGCs in the simultaneous transfection group increased. Shen (7) observed the protective effect of ultrasonic microbubbles combined with mouse nerve growth factor (mNGF) on rabbit hypertension optic nerve damage, and the results showed that RGCs counts in the mNGF and ultrasound microbubble group were significantly higher than those in the hypertension group and mNGF only injection group $(\mathrm{P}<0.05)$. The rabbit retinal structure of each layer in the $\mathrm{mNGF}$ and ultrasound microbubble group was clearer and fuller than that in the hypertension and mNGF injection only groups. The improvement of optic nerve ultrastructure in the mNGF and ultrasound microbubble group was significantly better than those in the hypertension and mNGF injection only group. 
Currently, Glaucomatous optic nerve damage is explained by several theories, including mechanical stress theory and vascular ischemia theory. In these theories, intraocular pressure is currently recognized as the most important risk factor, and had the most clinical intervention. And there are other influencing factors such as autoimmunity, nerve element, genetic element. With the development of researches on the mechanism of optic nerve damage in glaucoma, the progress of glaucoma gene screening and stem cell research, the emergence of neuroprotective drugs, the new trend of optic nerve protection showed up, such as promoting the early repair of retinal nerve DNA, inhibiting the activation of retinal glial cells, improving the connection between retinal neurons and upper neurons, gene therapy and stem cell therapy, etc.

\section{Conclusions}

In summary, ultrasound microbubbles combined with GSTT have a significant protective effect on optic nerve damage in rabbits with high intraocular pressure, which may be related to the changed content of GSTT in rabbit retina. The specific mechanism needs further study. The technique of ultrasound microbubbles is safe, controllable, nonimmunogenic, and repeatable. It offers a novel approach in the administration of neuroprotective drugs.

\section{Acknowledgments}

Funding: This work was supported by supported by Shenzhen Key Medical Discipline Construction Fund (No. SZXK037), Sanming Project of Medicine in Shenzhen (No. SZSM201812091), National Nature Science Foundation of China (No. 82070961), International Science and Technology Cooperation Research Project of Shenzhen Science and Technology Innovation Committee (GJHZ20190929145402153), the Science and Technology Innovation Committee of Shenzhen (No. JCYJ20190807153005579), and the Guangdong Basic and Applied Basic Research Foundation (No. 2019A1515011234).

\section{Footnote}

Reporting Checklist: The authors have completed the ARRIVE reporting checklist. Available at https://dx.doi. org/10.21037/atm-21-4230

Data Sharing Statement: Available at https://dx.doi. org/10.21037/atm-21-4230

Conflicts of Interest: All authors have completed the ICMJE uniform disclosure form (available at https://dx.doi. org/10.21037/atm-21-4230). The authors have no conflicts of interest to declare.

Ethical Statement: The authors are accountable for all aspects of the work in ensuring that questions related to the accuracy or integrity of any part of the work are appropriately investigated and resolved. Experiments were performed under a project license granted by the Ethics Committee of Medical School of Shenzhen University, in compliance with the International Association of Veterinary Editors' Consensus Author Guidelines on Animal Ethics and Welfare for the care and use of animals.

Open Access Statement: This is an Open Access article distributed in accordance with the Creative Commons Attribution-NonCommercial-NoDerivs 4.0 International License (CC BY-NC-ND 4.0), which permits the noncommercial replication and distribution of the article with the strict proviso that no changes or edits are made and the original work is properly cited (including links to both the formal publication through the relevant DOI and the license). See: https://creativecommons.org/licenses/by-nc-nd/4.0/.

\section{References}

1. Russo R, Nucci C, Corasaniti MT, et al. Autophagy dysregulation and the fate of retinal ganglion cells in glaucomatous optic neuropathy. Prog Brain Res 2015;220:87-105.

2. Shahid M, Riaz M, Talpur MM, et al. Phytopharmacology of Tribulus terrestris. J Biol Regul Homeost Agents 2016;30:785-8.

3. Wang Y, Guo W, Liu Y, et al. Investigating the Protective Effect of Gross Saponins of Tribulus terrestris Fruit against Ischemic Stroke in Rat Using Metabolomics and Network Pharmacology. Metabolites 2019;9:240.

4. Chauhdary Z, Saleem U, Ahmad B, et al. Neuroprotective evaluation of Tribulus terrestris $\mathrm{L}$. in aluminum chloride induced Alzheimer's disease. Pak J Pharm Sci 2019;32:805-16.

5. Timbie KF, Mead BP, Price RJ. Drug and gene delivery across the blood-brain barrier with focused ultrasound. J Control Release 2015;219:61-75.

6. Wang L, Cao T, Chen H. Treatment of glaucomatous 
optic nerve damage using ginsenoside $\mathrm{Rg} 1$ mediated by ultrasound targeted microbubble destruction. Exp Ther Med 2018;15:300-4.

7. Shen X, Huang L, Ma D, et al. Ultrasound Microbubbles Enhance the Neuroprotective Effect of Mouse Nerve Growth Factor on Intraocular Hypertension-Induced Neuroretina Damage in Rabbits. J Ophthalmol 2016;2016:4235923.

8. Nio AQX, Faraci A, Christensen-Jeffries K, et al. Optimal Control of SonoVue Microbubbles to Estimate Hydrostatic Pressure. IEEE Trans Ultrason Ferroelectr Freq Control 2020;67:557-67.

9. Liu Y, Gong Y, Xie W, et al. Microbubbles in combination with focused ultrasound for the delivery of quercetinmodified sulfur nanoparticles through the blood brain barrier into the brain parenchyma and relief of endoplasmic reticulum stress to treat Alzheimer's disease. Nanoscale 2020;12:6498-511.

10. Lin CY, Lin YC, Huang CY, et al. Ultrasound-responsive neurotrophic factor-loaded microbubble- liposome complex: Preclinical investigation for Parkinson's disease treatment. J Control Release 2020;321:519-28.

11. Zhu $W, \mathrm{Du} Y$, Meng $\mathrm{H}$, et al. A review of traditional pharmacological uses, phytochemistry, and pharmacological activities of Tribulus terrestris. Chem Cent J 2017;11:60.

12. Ppoyil SBT, Rasheedpalakkott A, Ksiksi T. Tribulus arabicus and Tribulus macropterus are Comparable to Tribulus terrestris: An Antioxidant Assessment. Current Bioactive Compounds 2017;13:752-5.

13. Huang L, Wang L, Ying F, et al. The protective effect of Gross Saponin of Tribulus Terrestris on rat retinal

Cite this article as: Shen X, Guo J, Fan N, Lai M, Huang L, Wang J, Li Q. Protective effect of ultrasound microbubble combined with gross saponins of tribulus terrestris on glaucomatous optic nerve damage. Ann Transl Med 2021;9(18):1436. doi: 10.21037/atm-21-4230 ganglion cells in vitro. Journal of Traditional Chinese Ophthalmology 2008;18:89-91.

14. Li N, Huang L, Zeng P, et al. Protective effect of gross saponins from tribulus terrestris $\mathrm{L}$ on retinal ganglion cells in rabbits with chronic high intraocular pressure. International Journal of Ophthalmology 2010;10:459-61.

15. McMahon D, Oakden W, Hynynen K. Investigating the effects of dexamethasone on blood-brain barrier permeability and inflammatory response following focused ultrasound and microbubble exposure. Theranostics 2020;10:1604-18.

16. Wang Y, Li X, Liu L, et al. Tissue Targeting and Ultrasound-Targeted Microbubble Destruction Delivery of Plasmid DNA and Transfection In Vitro. Cell Mol Bioeng 2020;13:99-112.

17. Kopechek JA, Mc'Tiernan CF, Chen X, et al. Ultrasound and Microbubble-targeted Delivery of a microRNA Inhibitor to the Heart Suppresses Cardiac Hypertrophy and Preserves Cardiac Function. Theranostics 2019;9:7088-98.

18. Lin YC, Chen HC, Chen HK, et al. Ultrastructural Changes Associated With the Enhanced Permeability of the Round Window Membrane Mediated by Ultrasound Microbubbles. Front Pharmacol 2020;10:1580.

19. Fu Y, Liu S, Wang Z, et al. Protection of retinal ganglion cells from optic nerve injury by brain-derived neurotrophic factor transfected through microbubble contrast agent into the retina and visual cortex. Chinese Journal of Ocular Fundus Diseases 2011;27:65-9.

(English Language Editor: B. Meiser) 